\title{
A Study and Analysis of Improved Binary Pattern Technique in Dynamic Images
}

\author{
R.Thirumalaisamy ${ }^{\text {a }}$, Dr.S.Kother Mohideen ${ }^{\text {b }}$
}

\author{
${ }^{a}$ Ph.D. Research Scholar,Department of Information Technology, Sri Ram Nallamani Yadava College of \\ Arts and Science, Tenkasi, (Affiliated to Manonmaniam Sundaranar University, Tirunelveli - 627012 , \\ Tamilnadu,India) \\ ${ }^{\mathrm{b}}$ HOD \& Research Center Head,Department of Information Technology, Sri Ram Nallamani Yadava \\ College of Arts and Science, Tenkasi, (Affiliated to Manonmaniam Sundaranar University, Tirunelveli - \\ 627 012, Tamilnadu,India)
}

\begin{abstract}
A dynamic image has a distinct quantity of object movement from one to another. It can be any object such as a car, person, an object moving from one point $\mathrm{X}$ to another point $\mathrm{Y}$. Image consists of a sense of movement. Applications of object tracking are biometrics tracking, AR uses, video surveillance, passage monitoring, vehicle navigation, etc. Challenges in tracking multifaceted objects are fast movement, geometric conversion, blurring, messy background, artifacts, etc. To resolve this problem by merge all small features with nearby texture features. Texture feature describes the plane space and configuration of an area. A mixture of color and texture feature improves the object details and to increase the strength of the object's illustration. In Existing methods such as binary pattern method all object features are removed, so it is difficult to predict the exact pixel movement. The proposed method of improved binary pattern is also tracking the small changes in the pixel difference in one frame to other. Compared with the existing algorithms, IBP method measures the spatial arrangement of local image texture which reduces the overall processing cost and improves the strength of objective image. To track the similarities and difference of the object in each and every frame efficiently and effectively Improved Local Binary Pattern tracking algorithm was proposed. This proposed technique is an effective way to analysis complicated real time situations compared with other methods.
\end{abstract}

Keywords: Binary pattern, Dynamic Images, Features, Improved Binary Pattern, Object Tracking, Texture

\section{Introduction}

Various algorithms related to object tracking methods such as point detection and tracking are surveyed. Tracking methods needs a set of exclusive features to characterize each followed object. Choose the correct features is very much connected to the thing. Andersson et.al [1] proposed a KF based algorithm considering Gaussian and linear problems to follow one's pose in interactive computer graphics. Gaussian and linear systems are studied based on particle filter-based approaches [2,3]. Other common trackers used are comprises of optical flow [4], multiple hypothesis [5, 6], and kernel [7,8]. Recently, João F. Henriques et al. [9] proposed KT algorithm is high speed of track having linear correlation filters are used. Kernel algorithms are challenging problems such as lighting change, object deblurs, noises, rapid movement, and similarly colored backgrounds. MS algorithm can hold object limited changes and background subtraction. It shows a non parametric prototype matching follow algorithm. It employs the histogram as the objective representation and the relationship calculation. The object position in a frame is founded by repetitive process [10]. The performance calculation is resolute by the resemblance computation and the objective feature. The position of the object, similarity measures, such as standardized cross section, intersection distance was explained by the Bhattacharyya [11].yang [12] et.al explains the Incremental knowledge for strong illustration Tracking algorithms which is faster method of track the moving object.

\section{Binary Pattern Technique}

In Binary pattern method, Let us consider $3 \times 3$ dimensions of pixel block of an image. All pixels values such that nearby pixels in the under block are compared with threshold by its center value either 1 or 0 . ILBP descriptor produces 256 texture patterns and illumination non changes quality features. The operator uses the binary values of all the pixels in dimensions to give exact details to the central pixel. 


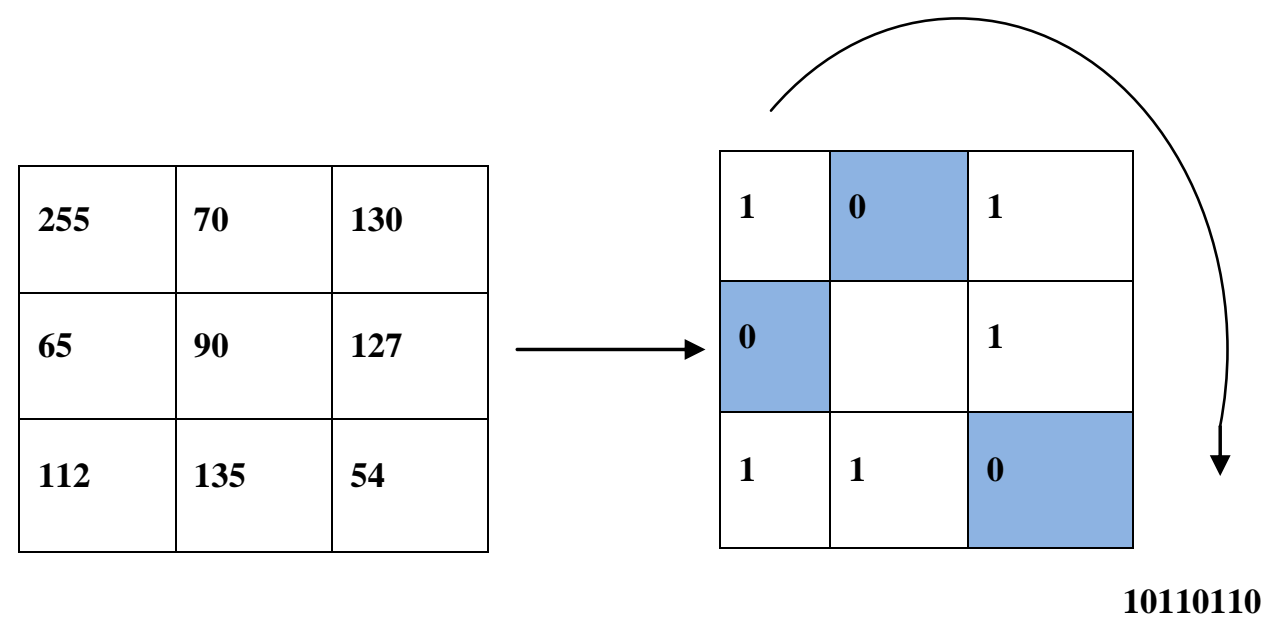

Threshold

Figure 1. Basic BP

BINARY CODE 10110110

DECIMAL NUMBER 182

Let consider middle pixel value as threshold [8] compared each and every pixel value in the $3 \mathrm{x} 3$ block with center pixel value. for example center pixel value is $\mathrm{P}(\mathrm{x})$, compare $\mathrm{P}(\mathrm{x})$ with first row value as $\operatorname{Ri}(\mathrm{x})$, if $\operatorname{Ri}(\mathrm{x})>\mathrm{P}(\mathrm{x}) \operatorname{mark}$ as 1 , then check if $\operatorname{Ri}(x)<P(x)$ mark as 0 , then convert the pixel value into binary to process.

The texture model $\mathrm{BP}_{\mathrm{N}, \mathrm{R}}$ is expressed as

$$
\mathrm{BP}_{\mathrm{N}, \mathrm{R}}=\sum_{\mathrm{i}=0}^{\mathrm{N}-1} \mathrm{M}\left(\mathrm{T}_{\mathrm{i}}-\mathrm{T}_{\mathrm{c}}\right) 2^{\mathrm{i}}, \mathrm{K}(\mathrm{x})=1, \mathrm{x} \geq 0,
$$

Where, $\mathrm{N}$ denotes neighbours pixels numbers, its radius of the middle pixel is represented as $\mathrm{R}$, where represents the grey scale value of the middle pixel value and $T_{c}$ neighbours pixels numbers with initial value of $i=0$ to final value $\mathrm{N}-1$ and sign value represented as $\mathrm{K}(\mathrm{x})$. The operator mark the pixels of an image by threshold value in $3 \times 3$ dimensions nearby value of every pixel compared with the center pixel value and give the end result as binary value. LBP operator can be extended to different size of neighborhood pixels. A circular neighborhood value denoted by (N.R). Where $\mathrm{N}$ is the sampling number and $\mathrm{R}$ is the radius of circle. The edge of this circle area is compared with the value threshold pixel value. To obtain the value of sample pixels in the neighborhood of any number of pixels and its radius are processed by bilinear method. For neighborhood values (N, R) is used. Figure shows neighbor sets for different values of $\mathrm{N}$ and $\mathrm{R}$.

\section{Improved Binary Pattern Technique}

In Improved Binary pattern techniques[6] are bitwise changes from 0 to 1 in circular demonstration of the BP code always attend a bitwise change from 1 to 0 and vice versa. The Improved $B P$, can only get an even number between 0 to $\mathrm{N}$ because it corresponds to the number of spatial transitions in the BP code is denoted as circular. In conventional Binary pattern $\mathrm{N}$, the dissimilarity between middle pixel and neighbor pixels in the local area is returned in the structure of encoding. The middle pixel value is lower; the spatial connection of the whole pixel is ignored. In order to resolve this problem, the proposed IBP technique increases the quantity of the middle pixel value in sampling regions and emphasizes the connection between the sampling region and the whole image. Calculate the dissimilarity between the adjacent pixel values in the crosswise situation and evaluate the value of the middle pixel with all the pixel values in the region 

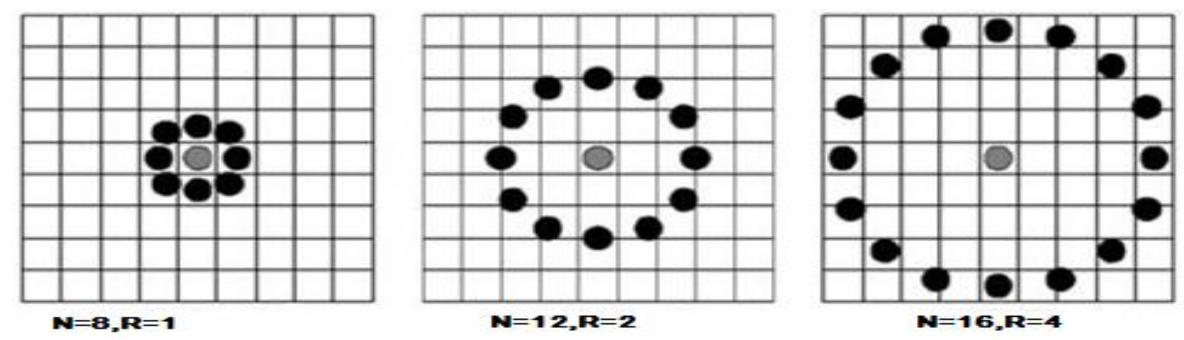

Figure 2.Centroid Pixel Tracking Radius

The texture model IBP ${ }_{\mathrm{N}, \mathrm{R}}$ is expressed as

$$
\begin{aligned}
& \mathrm{IBP}_{\mathrm{N}, \mathrm{R}}=\sum_{i=\mathbf{0}}^{N-\mathbf{1}} \mathrm{M}(\mathrm{Ti}-\mathrm{Tc}) 2 \mathbf{i}-\mathbf{1}, \quad \mathrm{U}(L B P N, R) \leq 2 \\
& \mathbf{M}-\mathbf{1}+\mathbf{U} / \mathbf{2}, \quad \mathbf{U}(\mathbf{L B P N}, \mathbf{R})>2
\end{aligned}
$$

Where, $\mathrm{N}$ denotes neighbours pixels numbers, its radius of the middle pixel is represented as $\mathrm{R}$, where represents the grey scale value of the middle pixel value and $T_{c}$ neighbours pixels numbers with initial value of $i=0$ to final value $\mathrm{N}-1$.

\section{Algorithm for IBP}

1. Set the central threshold pixel value $\mathrm{T}$ as the initial position.

2. Calculate the values $\operatorname{Ri}(\mathrm{x})$ of each pixel of the region

3. If $\operatorname{Ri}(x)>P(x)$ mark as 1

4. Otherwise If $\operatorname{Ri}(\mathrm{x})<\mathrm{P}(\mathrm{x})$ mark as 0

5. Calculate the target location $\mathrm{i}=\mathrm{i}+1$

6. Adjust the scale of the objective region.

7. Examine the next block of the sequence and repeat Step 1

\begin{tabular}{|l|l|l|}
\hline 1 & 0 & 1 \\
\hline 0 & 0 & 0 \\
\hline 1 & 1 & 1 \\
\hline
\end{tabular}

\begin{tabular}{|l|l|l|}
\hline 1 & 1 & 1 \\
\hline 1 & 1 & 1 \\
\hline 0 & 0 & 0 \\
\hline
\end{tabular}

\begin{tabular}{|l|l|l|}
\hline 1 & 0 & 1 \\
\hline 0 & 1 & 0 \\
\hline 1 & 0 & 1 \\
\hline
\end{tabular}

Figure 3. Non-Uniform Pixel formation of L, T and Cross section 


\section{Result Analysis}

The performance of IBP technique has been verified by widespread [15] set of experiments. Proposed method compared with many other tracking methods such as Mean shift tracking, kernel etc. When input frame of the compressed video is given as input. It creates a structured for way based on ILBP. In evaluation, improved binary pattern are not changes in same gray scale dissimilarity and rotation [11] conversion. Binary pattern allocates non-uniform patterns to a various pixel values, whereas improved binary pattern allocates different labels according to their uniformity standards. It determines collection of local primitives such as lines, T-junctions and cross intersections, which are unobserved by binary pattern.

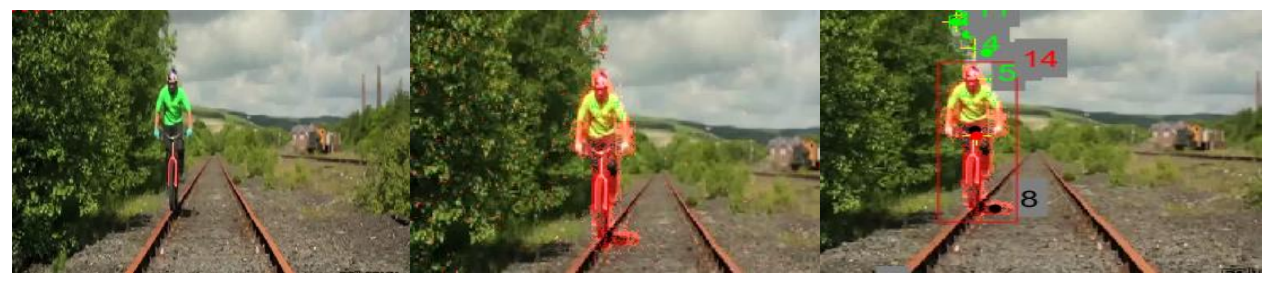

Figure 4. Input Image (left), Feature Selection (Right)

To detect the objective area after the tracking procedure are finished. When compared with LBP method, the IBP method has a best tracking performance over the full sequence. This method is can track the moving object very efficiently and quickly. Object movement tracking information [17] was extracted from the bit stream. The below figure shows the movement of pixel was plotted in spatial domain. Figure 5, shows the pixel values in an object, different pixel value position are identified from one point to another from each and every frame by comparing present frame with reference frame and it will be displayed as pixel plotter.

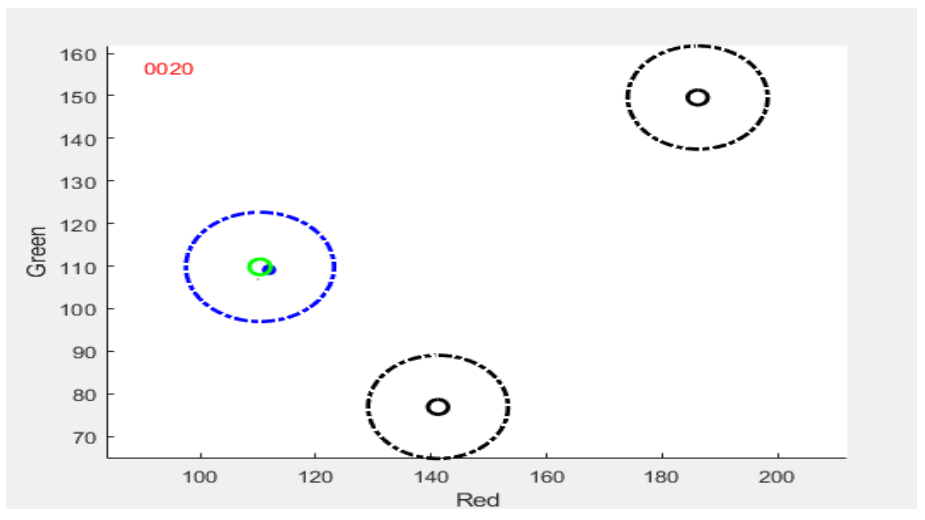

Figure 5. Moving Pixel Plotter

The below table 1 shows the evaluation of various training angle of binary pattern and improved binary pattern techniques. Thus, experimental results [11] on dataset RotInv [21] shows that Improved Binary Pattern is more discriminative than conventional Binary Pattern method even though both invariant to rotation conversion. The data set was employed earlier to carry out rotation invariant [12] texture classification. Textures are offered at different angles $\left(0^{\circ}, 30^{\circ}, 45^{\circ}, 60^{\circ}, 90^{\circ}, 120^{\circ}\right.$ and $\left.150^{\circ}\right)$. 
Table 1: Comparison of data training angle of BP and IBP

\begin{tabular}{|c|c|c|c|c|c|c|}
\hline \multirow{2}{*}{$\begin{array}{l}\text { Training } \\
\text { Angle in } \\
\text { degrees }\end{array}$} & \multicolumn{3}{|c|}{ BP(N,R) } & \multicolumn{3}{c|}{ IBP(N,R) } \\
\cline { 2 - 7 } & $\mathbf{( 8 , 1 )}$ & $\mathbf{( 1 2 , 2 )}$ & $\mathbf{( 1 6 , 4 )}$ & $\mathbf{( 8 , 1 )}$ & $\mathbf{( 1 2 , 2 )}$ & $(\mathbf{1 6 , 4 )}$ \\
\hline 0 & 68.2 & 98.2 & 96.3 & 68.2 & 98.2 & 98.2 \\
\hline 30 & 76.5 & 78.4 & 90.5 & 86.1 & 98.3 & 98.3 \\
\hline 45 & 78.5 & 96.5 & 97.5 & 88.5 & 99.3 & 97.5 \\
\hline 60 & 79.3 & 85.8 & 89.2 & 74.1 & 98.3 & 99.3 \\
\hline 90 & 69.7 & 98.5 & 97.4 & 79.3 & 99.5 & 99.5 \\
\hline 120 & 76.4 & 89.5 & 98.7 & 86.4 & 98.7 & 98.7 \\
\hline 150 & 74.1 & 93.2 & 90.2 & 89.2 & 99.6 & 99.6 \\
\hline Mean & 74.7 & 91.4 & 94.3 & 81.7 & 98.8 & 98.7 \\
\hline
\end{tabular}

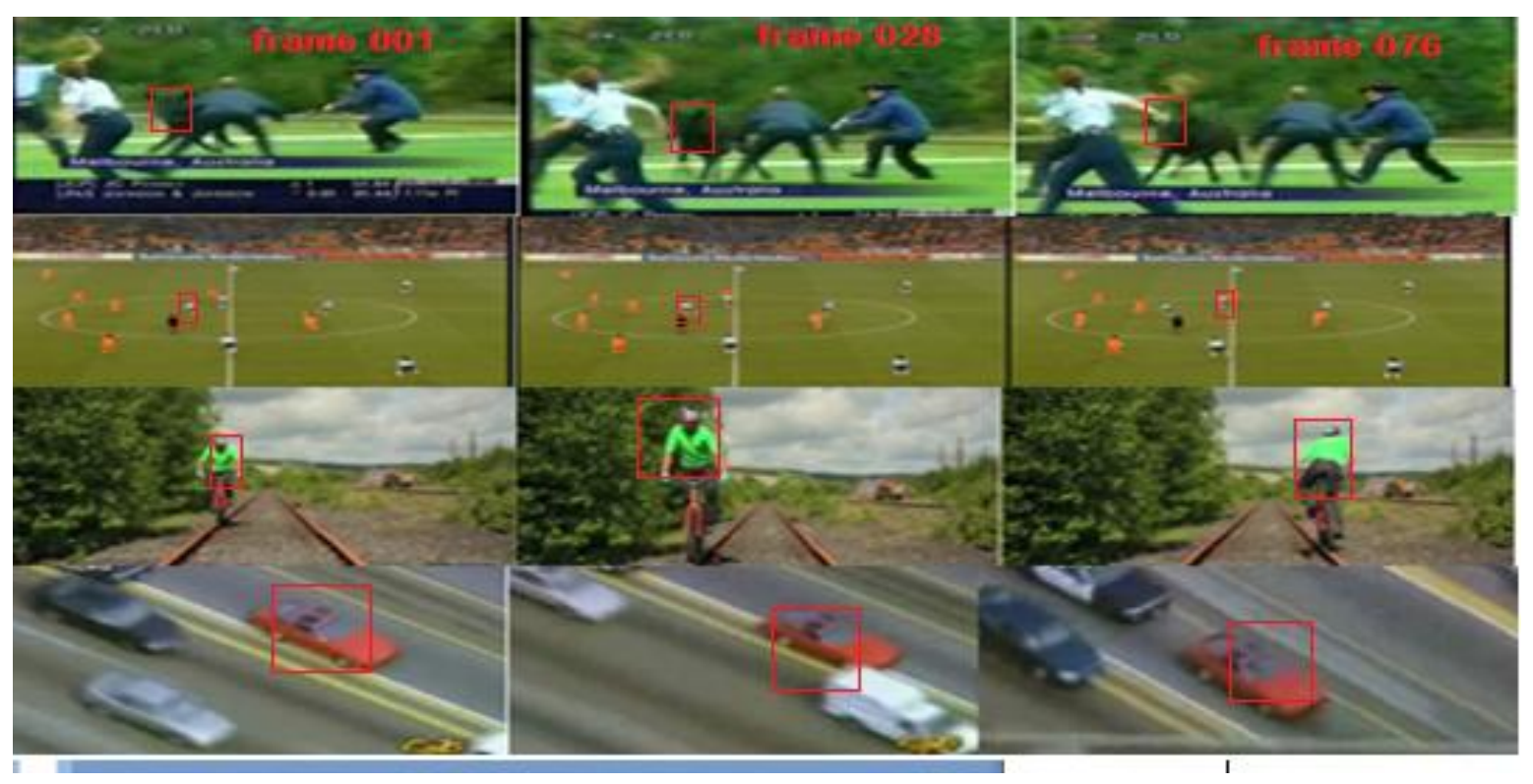

Figure 6. Various Dataset Images

Figure 6, shows the information of the moving objects is tracked by various [20][21] datasets. When the object such as a cow in the upper picture moving from one position to another position in each and every frame, it was accurately tracking the various pixel movement by the proposed method, the same procedure was followed by various datasets such as cycling, football, car, etc. Table 2 demonstrates various datasets such as cycling, car, cow, and football image frames are given as input and their results of binary pattern and improved binary pattern are analyzed. 
Table 2. BP and IBP Result Comparison

\begin{tabular}{|l|l|l|l|}
\hline Sequences & Frames & $\mathbf{B P}(\mathbf{N}, \mathbf{R})$ & $\mathbf{I B P ( N , R )}$ \\
\hline Cycling & 1000 & $0.954 / 0.884 / 0.918$ & $0.954 / 0.884 / 0.918$ \\
\hline Cow & 850 & $0.97 / 0.97 / 0.97$ & $0.97 / 0.97 / 0.97$ \\
\hline Car & 407 & $1.0 / 1.0 / 1.0$ & $1.0 / 1.0 / 1.0$ \\
\hline Football & & & $0.97 / 0.97 / 0.97$ \\
\hline
\end{tabular}

\section{Conclusion}

The proposed binary pattern tracking algorithms are efficient than other precise tracking algorithms, especially low computational resources and limited bandwidth. This method is a novel approach to track an object accurately at minimum storage requirements.IBP algorithm reaches high efficiency of pixel-domain tracking and reliably operates on real datasets consuming very much less data processing time. The proposed algorithm is an effective way to track real-time situations compared with other state-of-the-art methods. Finally the proposed improved binary pattern methods are tested with widespread dataset and results are analyzed. The IBP operator identifies the main group of local primitives from non-uniform patterns and thus the IBP feature has significant discriminative capability than conventional Binary patterns.

\section{References}

[1] J. Andersson, L. Baresi, N. Bencomo, R. Lemos, A. Gorla, P. Inverardi and T. Vogel, "Software Engineering Processes for [1] Welch G., Bishop G. SCAAT: Incremental Tracking with Incomplete Information; Proceedings of the 24th Annual Conference on Computer Graphics and Interactive Techniques; Los Angeles, CA, USA. 3-8 August 1997; New York, NY, USA: New York, NY, USA: ACM Press; Addison-Wesley Publishing Co.; 1997. pp. 333-344.

[2] [ Isard M., Blake A. Condensation - Conditional Density Propagation for Visual Tracking. Int. J. Comput. Vis. 1998;29:5-28..

[3] Choo K., Fleet D. People tracking using hybrid Monte Carlo filtering; Proceedings of the Eighth IEEE International Conference on Computer Vision; Vancouver, BC, Canada. 7-14 July 2001; pp. 321-328.

[4] Lucas B.D., Kanade T. An iterative image registration technique with an application to stereo vision; Proceedings of the IJCAI'81 Proceedings of the 7th international joint conference on Artificial intelligence; Vancouver, BC, Canada. 24-28 August 1981; pp. 674-679.

[5] Reid D. An algorithm for tracking multiple targets. IEEE Trans. Autom. Control. 1979;24:843-854.

[6] Cox I., Hingorani S. An efficient implementation of Reid's multiple hypothesis tracking algorithm and its evaluation for the purpose of visual tracking. IEEE Trans. Pattern Anal. Mach. Intell. 1996;18:138-150.

[7] Comaniciu D., Ramesh V., Meer P. Kernel-based object tracking. IEEE Trans. Pattern Anal. Mach. Intell. 2003;25:564-577.

[8] Jepson A., Fleet D., El-Maraghi T. Robust online appearance models for visual tracking. IEEE Trans. Pattern Anal. Mach. Intell. 2003;25:1296-1311.

[9] Henriques J.F., Caseiro R., Martins P., Batista J. High-Speed Tracking with Kernelized Correlation Filters. IEEE Trans. Pattern Anal. Mach. Intell. 2015;37:583-596..

[10] Comaniciu D., Ramesh V., Meer P. Real-time tracking of non-rigid objects using mean shift; Proceedings of the Computer Vision and Pattern Recognition; Hilton Head Island, SC, USA. 15 June 2000; pp. 142-149.

[11] T.Ojala, Matti Pietikainen, and Topi Maenpaa,"Multiresolution Gray-Scale and Rotation Invariant Texture Classification with Local Binary Patterns", IEEE Transactions on Pattern Analysis and Machine Intelligence, vol. 24 ,no.7, July 2002. 
[12] D. Ross, J. Lim, R. Lin, and M. Yang, “Incremental Learning for Robust Visual Tracking," International Journal of Computer Vision, vol. 77, pp. 125-141, Aug. 2007.

[13] R. Collins, Y. Liu, and M. Leordeanu, "Online Selection of Discriminative Tracking Features," IEEE Transactions on Pattern Analysis and Machine Intelligence, vol. 27, no. 10, pp. 1631-1643, 2005.

[14] S. Avidan, "Ensemble Tracking," IEEE Transactions on Pattern Analysis and Machine Intelligence, vol. 29, no. 2, pp. 261-271, 2007.

[15] B. Babenko, M.-H. Yang, and S. Belongie, "Visual Tracking with Online Multiple Instance Learning," Conference on Computer Vision and Pattern Recognition, 2009.

[16] Matusek F, Sutor S, Kraus K, et al. "NIVSS: a nearly indestructible video surveillance system", Proc of the 3rd International Conference on Internet Monitoring and Protection. Washington DC: IEEE Computer Society, 2008:98-102.

[17] Zdenek Kalal, Krystian Mikolajczyk, and Jiri Matas, "Forward-Back Error: Automatic Detection of Tracking Failures", International Conference on Pattern Recognition, 23-26 August, 2010, Istanbul, Turkey.

[18] Zdenek Kalal, Krystian Mikolajczyk, and Jiri Matas “Tracking-Learning-Detection”, IEEE Transactions on Pattern Analysis and Machine Intelligence, vol.6, no.1, JANUARY 2010.

[19] Zhou Xin, Qian Qiumeng, Ye Yongqiang, Wang Congqing Improved TLD visual target tracking algorithm [J]. Journal of Image and Graphics,2013,18(9):1115-1123.(in Chinese)

[20] Teng Fei,Robust Inland Waterway Ship Tracking via Hybrid TLD and Kalman Filter. Advanced Materials Research,2014, $1037: 373-377$

[21] Xiao Qingguo, Ye Qingwei, Zhou Yu, Wang Xiaodong. Long-term video tracking algorithm of optimized TLD based on mean-shift .Application Research of Computer, 2015,32(3):925-928.

[22] Yu Genzhen. Research on multi-target tracking based on TLD algorithm [D]. Master thesis, Xiamen University, 2012.

[23] Di Huang, Caifeng Shan, "Local Binary Patterns and Its Application to Facial Image Analysis: A Survey", IEEE Transactions on Systems, Man, and Cybernetics-part C: Applications and Reviews, vol.41, no.6, November, 2011.

[24] T.Ojala, Matti Pietikainen, and Topi Maenpaa,"Multiresolution Gray-Scale and Rotation Invariant Texture Classification with Local Binary Patterns", IEEE Transactions on Pattern Analysis and Machine Intelligence, vol. 24 ,no.7, July 2002.

[25] D. Ross, J. Lim, R. Lin, and M. Yang, “Incremental Learning for Robust Visual Tracking,” International Journal of Computer Vision, vol. 77, pp. 125-141, Aug. 2007.

[26] R. Collins, Y. Liu, and M. Leordeanu, "Online Selection of Discriminative Tracking Features," IEEE Transactions on Pattern Analysis and Machine Intelligence, vol. 27, no. 10, pp. 1631-1643, 2005.

[27] S. Avidan, "Ensemble Tracking," IEEE Transactions on Pattern Analysis and Machine Intelligence, vol. 29, no. 2, pp. 261-271, 2007.

[28] B. Babenko, M.-H. Yang, and S. Belongie, "Visual Tracking with Online Multiple Instance Learning," Conference on Computer Vision and Pattern Recognition, 2009.

[29] Q. Yu, T. B. Dinh, G. Medioni, "Online tracking and reacquisition using co-trained generative and discriminative trackers” European Conference on Computer Vision, 2008.

[30] H. Grabner and H. Bischof, "On-line boosting and vision," Conference on Computer Vision and Pattern Recognition, 2006.

[31] A. Saffari, C. Leistner, J. Santner, M. Godec, and H. Bischof, "Online Random Forests,” Online Learning for Computer Vision Workshop, 2009. 